\title{
INVESTIGATION OF CONVERSION VARIOUS ILIDENMALONONITRILES
}

\author{
A.M. Maharramov, F.N. Naghiyev, A.R. Asgarova, A.G. Rahimova, \\ M.A. Akhundova, I.G. Mamedov \\ Baku State University, \\ Z.Khalilov str. 23, AZ 1148 Baku, Azerbaijan \\ e-mail: farid.orgchemist@gmail.com
}

\begin{abstract}
Appropriate derivatives of spiropiridine have first been obtained by means of one-stage condensation of isatilidenmalononitriles, malononitriles and 2-tiophenmethylamine (or furfurilamine). Besides, by interaction of benzylidenemalononitriles with thiosemicarbazide or 2,4-dinitrophenylhydrazines appropriate Schiff-bases obtained. Structures of synthesized compounds obtained confirmed through ${ }^{1} \mathrm{H}$ and ${ }^{13} \mathrm{C} N \mathrm{~N}$ R spectroscopy methods.
\end{abstract}

Keywords: isatilidenemalononitrile, malononitrile, amines, thiosemicarbazide, hydrazine

\section{INTRODUCTION}

Benzylidenemalononitriles as members of ilidenes class are used as synthones in the synthesis of biologically active compounds, such as $4 H$-pyrane, substituted pyridines, pyrazoles, imidazopyridine derivatives. It has been widely investigated anticoagulant, anticancer, spasmolitic, anti-anafilactic properties of $4 H$-pyranes and dihydropiridines<smiles>N#CC[O+]=C1COC(=O)C1</smiles>

Note that the simple synthesis has been carried out through the Michaels addition reaction of benzylidenemalononitriles and $\mathrm{N}, \mathrm{N}$-dialkylbarbituric acid in the presence of derivatives, which obtained by Michaels addition reactions of benzylidene malononitriles with methylenactive compounds [1-3].

Below-cited is data on effective onestage synthesis of spirooxindole derivatives by 3-component reaction of isatine, malononitrile and 1,3-dicarbonyl compounds in the aquous media and $10 \mathrm{~mol} \%$ L-prolin [4].<smiles>N#CC1=C(N)OC2=C(C(=O)OC2)C12C(=O)Nc1ccccc12</smiles>

molecular bromine and sodium ethylate by 75 95\% yield, 2-aryl-4,6,8-trioxo-5,7diazaspiro[2.5] octane-1,1-dicarbonitriles [5].<smiles>[R]COCC(C#N)C#N</smiles><smiles>CN1C(=O)CC(=O)N(C)C1=O</smiles><smiles>CN1C(=O)C(C(c2ccccc2)C(C#N)(C#N)C#N)C(=O)N(C)C1=O</smiles>

Of interest is data on obtaining corresponding enamines by means of benzylidene [6].

malononitriles reaction with primary amines 


\section{RESULTS AND DISCUSSION}

Also, the 3-component one-stage furfurilamine) in methanol and room condensation of isatilidenemalononitrile, temperature has first been analyzed.

malononitrile and 2-thiophenmethylamine (or
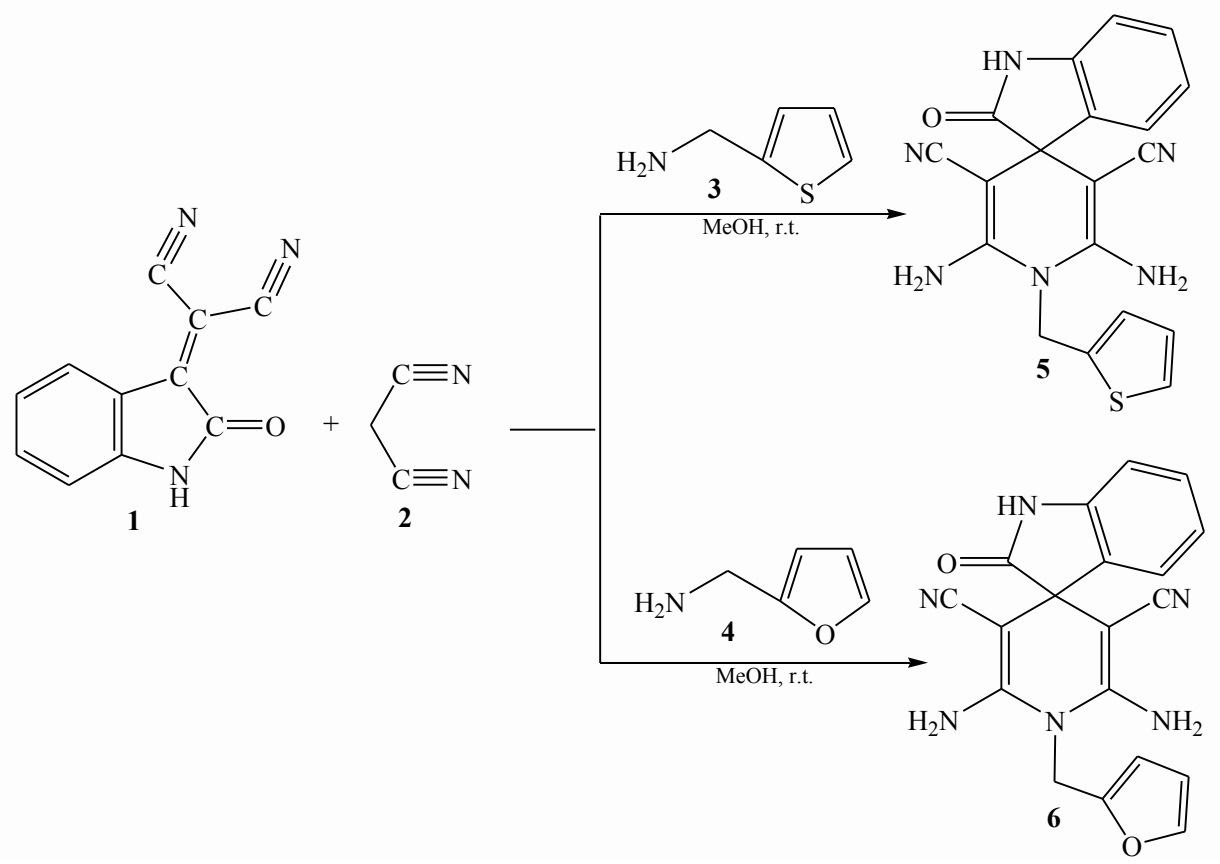

In terms of identical reaction conditions, the one-stage 3-component condensation reaction rile and thiosemicarbazide (or 2,4-dinitrophenylhydrazine) has been carried out to reveal between benzylidenemalononitrile, malononit- that malononitrile takes no part in the process.

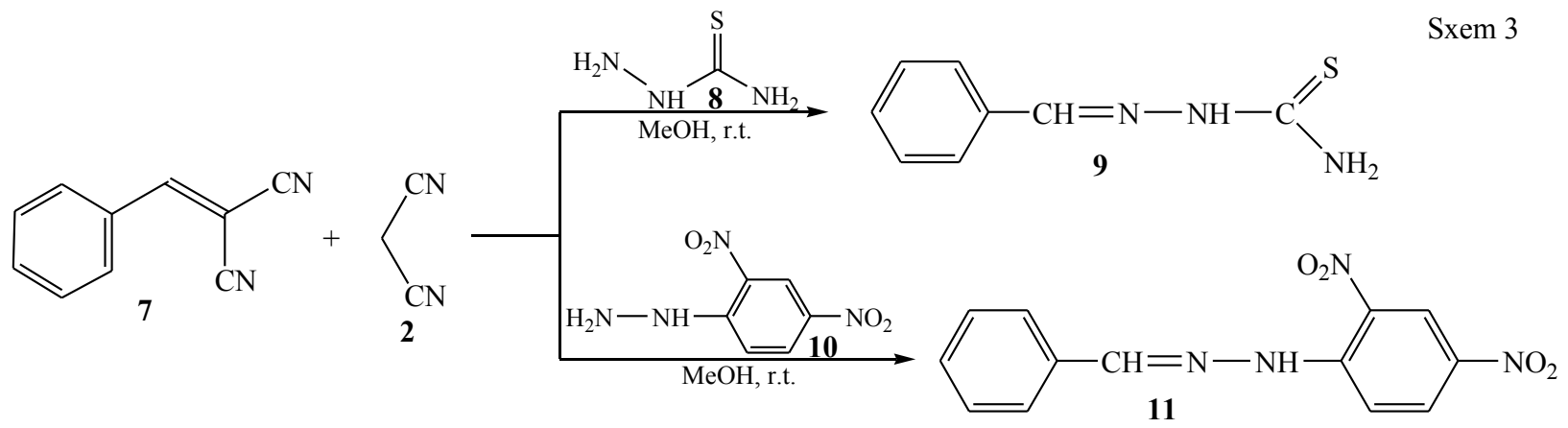

\section{EXPERIMENTAL PART}

All reagents were purchased from Merc and Fluca and used without cleaning. The melting points of compounds measured at STUART SPM30. Purity of synthesized compounds was complied with TLC, and structures confirmed on "Bruker300" NMR apparatus (300 and $75 \mathrm{MHz}$ ).

The general technique of obtaining spiroindolin-substituted pyridine derivatives is as follows: mixture of isatilidenemalononitrile $(4.3 \mathrm{mmol})$ and 
malononitrile ( $4.4 \mathrm{mmol})$ dissolved in $30 \mathrm{ml}$ of methanol was stirred up for 5-7 minutes, then 2-thiophenmethylamine $(4.4 \mathrm{mmol})$ (or furfurilamine) added and stirring continued. Reaction progress was tracked by TLC (EtOAc/n-hexane, 2:1) and reaction mixture kept quietly for 48-72 hours. The evaporation of solvent was followed by precipitation of crystals. Also, crystals were separated by filter paper and recrystalliized from ethanol (95\%) water mixture.

\section{2',6'-Diamino-2-oxo-1'-(thiophen-2-} ylmethyl)-1'H-spiro[indoline-3,4'-pyridine]3',5'-dicarbonitrile (5):

${ }^{1} \mathrm{H}$ NMR spektr (DMSO-d6), $\delta$, m.h.: $3.37 \mathrm{~s}\left(2 \mathrm{H}, \mathrm{CH}_{2} \mathrm{~N}\right), 3.88 \mathrm{~s}\left(4 \mathrm{H}, 2 \mathrm{NH}_{2}\right), 6.31-$ $8.13 \mathrm{~m}\left(7 \mathrm{H}, 4 \mathrm{CH}_{\text {arom }}+3 \mathrm{CH}=\right), 10.55 \mathrm{~s}(1 \mathrm{H}$, $\mathrm{NH}) .{ }^{13} \mathrm{C}$ NMR spektr (DMSO- $d 6$ ), $\delta$, m.h.: $43.24\left(\mathrm{CH}_{2} \mathrm{~N}\right), 54.52\left(\mathrm{C}_{\text {tert }}\right), 61.30\left(=\mathrm{C}_{\text {tert }}\right)$, $76.75 \quad\left(=\mathrm{C}_{\text {tert }}\right), \quad 109.99 \quad\left(\mathrm{CH}_{\text {arom }}\right), \quad 110.20$ $\left(\mathrm{CH}_{\text {arom}}\right), 116.34(\mathrm{CN}), 116.84(\mathrm{CN}), 122.12$ $\left(\mathrm{CH}_{\text {arom }}\right), \quad 122.80 \quad\left(\mathrm{CH}_{\text {arom }}\right), \quad 124.16 \quad\left(\mathrm{C}_{\mathrm{ar}}\right)$, $124.67 \quad\left(\mathrm{C}_{\mathrm{ar}}\right), \quad 126.57 \quad\left(\mathrm{CH}_{\text {arom }}\right), \quad 130.34$ $\left(\mathrm{CH}_{\text {arom }}\right), 130.66\left(\mathrm{CH}_{\text {arom }}\right), 159.60 \quad\left(=\mathrm{C}_{\text {tert }}\right)$, $160.23 \quad\left(=\mathrm{C}_{\text {tert }}\right), \quad 175.10 \quad\left(=\mathrm{C}_{\text {tert }}-\mathrm{S}\right), \quad 177.33$ $(\mathrm{CONH}) . \mathrm{T}_{\mathrm{mp}}=268^{\circ} \mathrm{C}$.

Found, \%: $60.90 \mathrm{C} ; 3.68 \mathrm{H}$. $\mathrm{C}_{19} \mathrm{H}_{14} \mathrm{~N}_{6} \mathrm{OS}$. Calculated, \%: $60.96 \mathrm{C} ; 3.74 \mathrm{H}$.

2',6'-Diamino-1'-(furan-2-ylmethyl)-

2-oxo-1'H-spiro[indoline-3,4'-pyridine]3',5'-dicarbonitrile (6):

${ }^{1} \mathrm{H}$ NMR spektr (DMSO-d6), $\delta$, m.h.: $3.36 \mathrm{~s}\left(2 \mathrm{H}, \mathrm{CH}_{2} \mathrm{~N}\right), 3.78 \mathrm{~s}\left(2 \mathrm{H}, \mathrm{NH}_{2}\right), 6.62-$ $7.26 \mathrm{~m}\left(7 \mathrm{H}, 4 \mathrm{CH}_{\text {arom }}+3 \mathrm{CH}=\right), 7.69 \mathrm{~s}(2 \mathrm{H}$, $\left.\mathrm{NH}_{2}\right), 10.56 \mathrm{~s}(1 \mathrm{H}, \mathrm{NH}) .{ }^{13} \mathrm{C}$ NMR spektr (DMSO-d6), $\delta$, m.h.: $54.56\left(\mathrm{C}_{\text {tert }}\right), 56.63$ $\left(\mathrm{CH}_{2} \mathrm{~N}\right), 60.71\left(=\mathrm{C}_{\text {tert }}\right), 76.85\left(=\mathrm{C}_{\text {tert }}\right), 109.96$ $\left(\mathrm{CH}_{\text {arom }}\right), \quad 110.18\left(\mathrm{CH}_{\text {arom }}\right), 116.32(\mathrm{CN})$, $116.83(\mathrm{CN}), \quad 122.08 \quad\left(\mathrm{CH}_{\text {arom }}\right), \quad 122.63$
$\left(\mathrm{CH}_{\text {arom }}\right), 124.17\left(\mathrm{C}_{\mathrm{ar}}\right), 124.41\left(\mathrm{C}_{\mathrm{ar}}\right), 126.62$ $\left(\mathrm{CH}_{\text {arom }}\right), 130.27$ ( $\left.\mathrm{CH}_{\text {arom }}\right), 130.65\left(\mathrm{CH}_{\text {arom }}\right)$, $159.57\left(=\mathrm{C}_{\text {tert }}\right), 160.18\left(=\mathrm{C}_{\text {tert }}\right), 175.07\left(=\mathrm{C}_{\text {tert }}{ }^{-}\right.$ O), $177.32(\mathrm{CONH}) . \mathrm{T}_{\mathrm{mp}}=246^{\circ} \mathrm{C}$.

Found, \%: $63.63 \quad \mathrm{C} ; 3.87 \mathrm{H}$. $\mathrm{C}_{19} \mathrm{H}_{14} \mathrm{~N}_{6} \mathrm{O}_{2}$. Calculated, \%: $63.69 \mathrm{C} ; 3.91 \mathrm{H}$.

General synthesis method of azometin derivatives: Mixture of benzylidenemalononitriles $(5.8 \mathrm{mmol})$ and malononitrile $(5.9 \mathrm{mmol}$ ) dissolved in $30 \mathrm{ml}$ of methanol and thiosemicarbazide $(5.9 \mathrm{mmol})$ (or 2,4-dinitrophenylhydrazine) was added and stirred for 14 hours. Reaction progress was tracked by TLC (EtOAc/n-hexane, 2:1). Then reaction mixture was kept quitely for $48-72$ hours. After solvent evaporating, crystals perticipated and separated by filter paper. Product was recrystallized in pure form.

2-Benzylidenehydrazine-1-carbothioamide (9):

${ }^{1} \mathrm{H}$ NMR spektr (DMSO- $\left.d 6\right), \delta$, m.h.: $7.39 \mathrm{t}\left(3 \mathrm{H}, 3 \mathrm{CH}_{\text {arom }}\right), 7.78 \mathrm{~d}(2 \mathrm{H}, 2 \mathrm{CH}$ arom $)$, $8.06 \mathrm{~s}\left(2 \mathrm{H}, \mathrm{NH}_{2}\right), 8.22 \mathrm{~s}(1 \mathrm{H}, \mathrm{CH}=), 11.45 \mathrm{~s}$ $(1 \mathrm{H}, \mathrm{NH}) .{ }^{13} \mathrm{C}$ NMR spektr (DMSO-d6), $\delta$, m.h.: $127.76\left(2 \mathrm{CH}_{\text {arom }}\right), 129.12\left(2 \mathrm{CH}_{\text {arom }}\right)$, $130.31\left(\mathrm{CH}_{\text {arom }}\right), 134.62\left(\mathrm{C}_{\mathrm{ar}}\right), 142.77(\mathrm{CH}=)$, $178.44(\mathrm{C}=\mathrm{S}) . \mathrm{T}_{\mathrm{mp}}=156^{\circ} \mathrm{C}$.

\section{1-Benzylidene-2-(2,4-dinitrophenyl)-} hydrazine (11):

${ }^{1} \mathrm{H}$ NMR spektr (DMSO- $d 6$ ), $\delta$, m.h.: $7.49 \mathrm{t}\left(3 \mathrm{H}, 3 \mathrm{CH}_{\mathrm{arm}}\right), 7.80 \mathrm{~d}\left(2 \mathrm{H}, 2 \mathrm{CH}_{\mathrm{arm}}\right), 8.10$ $\mathrm{d}\left(1 \mathrm{H}, \mathrm{CH}_{\mathrm{arm}}\right), 8.37 \mathrm{~d}\left(1 \mathrm{H}, \mathrm{CH}_{\mathrm{arm}}\right), 8.70 \mathrm{~s}(1 \mathrm{H}$, $\mathrm{CH}=), 8.86 \mathrm{~s}\left(1 \mathrm{H}, \mathrm{CH}_{\mathrm{arm}}\right), 11.65(1 \mathrm{H}, \mathrm{NH}) .{ }^{13} \mathrm{C}$ NMR spektr (DMSO-d6), $\delta$, m.h.: 117.32 $\left(\mathrm{CH}_{\text {arom }}\right), 123.49\left(\mathrm{CH}_{\text {arom }}\right), 127.85\left(\mathrm{CH}_{\text {arom }}\right)$, 129.45 ( $\left.\mathrm{CH}_{\text {arom }}\right), 129.96$ ( $\left.\mathrm{CH}_{\text {arom }}\right), \quad 130.22$ $\left(\mathrm{CH}_{\text {arom }}\right), 131.03\left(\mathrm{C}_{\mathrm{ar}}\right), 134.28\left(\mathrm{C}_{\mathrm{ar}}\right), 137.47$ $\left(\mathrm{C}_{\mathrm{ar}}\right), 145.05($ tert $-\mathrm{C}=), 149.88(\mathrm{CH}=\mathrm{N}) . \mathrm{T}_{\mathrm{mp}}=$ $241^{\circ} \mathrm{C}$.

\section{REFERENCE}

1. Bonsignore L., Loy G., Secci D., Calignano A. Synthesis and pharmacological activity of 2-oxo-(2H) 1-benzopyran-3carboxamide derivatives. European Journal of Medicinal Chemistry. 1993, vol. 28, no.6, pp. 517-520.

2. Muharrem Kaya, Ersin Demir \& Hatice Bekci. Synthesis, characterization and antimicrobial activity of novel xanthene sulfonamide and carboxamide derivatives. Journal of Enzyme Inhibition and Medicinal Chemistry. 2013, vol. 28, no. 5, pp. 885-893.

3. Ulloora, Ramakrishna Shabaraya, Rajesh Ranganathan, Airody Vasudeva Adhikari. Synthesis, anticonvulsant and antiinflammatory studies of new 1,4dihydropyridin-4-yl-phenoxyacetohydra- 
zones. European Journal of Medicinal Chemistry. 2013, vol. 70, pp. 341-349.

4. Yuling Li, Hui Chen, Chunling Shi, Daqing Shi, and Shunjun Ji. Efficient One-Pot Synthesis of Spirooxindole Derivatives Catalyzed by L-Proline in Aqueous Medium. J.Comb.Chem. 2010, vol. 12, no. 2, pp. 231-237.

5. Michail N. Elinson, Anatolii N.Vereshchagin, Nikita O.Stepanov, Tatiana A.Zaimovskaya, Valentina M. Merkulova, Gennady I. Nikishin. The first example of the cascade assembly of a spirocyclopropane structure: direct transformation of benzylidenemalononitriles and $N, N^{\prime}$-dialkylbarbituric acids into substituted 2-aryl4,6,8-trioxo-5,7-diazaspiro[2.5]octane-1,1dicarbonitriles. Tetrahedron Letters. 2010, vol. 51, no. 2, pp. 428-431.

6. Liqi Shi, Yanyan Fu, Chao He, Defeng Zhu, Yixun Gao, Yuerong Wang, Qingguo He, Huimin Cao and Jiangong Cheng. A mild and catalyst-free conversion of solid phase benzylidenemalononitrile/benzylidenemalonate to N-benzylidene-amine and its application for fluorescence detection of primary alkyl amine vapor. Chem. Commun. 2014, v.50, no. 7. pp. 872-874.

\title{
BOZI ILIDENMALONONITRILLORIN ÇEVRILMO REAKSIYYASININ TODQIQQI
}

\author{
A.M. Mohərromov, F.N. Nă̆ıyev, A.R. Osgarova, A.Q. Rəhimova, \\ M.O. Axundova, I.Q. Mommodov \\ Bakı Dövlat Universiteti \\ AZ 1148 Bakl, Z.Xəlilov küç., 23; e-mail: farid.orgchemist@gmail.com
}

Illk dafo olaraq izatilidenmalononitrillarin malononitril va 2-tiofenmetilamin (yaxud furfurilamin) ila birmarhalali üç-komponentli reaksiyası aparılmış va reaksiyadan uyğun spiropiridin töramalari alınmışdır. Hamçinin benzilidenmalononitrillarin tiosemikarbazid va ya 2,4-dinitrofenilhidrazin ila qarşıllqqu tasir reaksiyasından uyğun Şiff əsasının alındı̆̆ı müşahidə edilmişdir. Anınan birloşmələrin quruluşu ${ }^{1} \mathrm{H}$ və ${ }^{13} \mathrm{C}$ NMR spektroskopiyasının köməyila tasdiq olunmuşdur.

Açar sözlor: izatilidenmalononitril, malononitril, aminlar, tiosemikarbazid, hidrazin

\section{ИССЛЕДОВАНИЕ ПРЕВРАЩЕНИЯ НЕКОТОРЫХ ИЛИДЕНМАЛОНОНИТРИЛОВ}

\author{
А.М. Магеррамов, Ф.Н. Нагиев, А.Р. Аскерова, А.Г. Рагимова, \\ М.А. Ахундова, И.Г. Мамедов \\ Бакинский государственный университет \\ AZ 1148 Баку, ул. 3.Халилова, 23; e-mail:farid.orgchemist@gmail.com
}

\begin{abstract}
Впервые путем трехкомпонентной одностадийной конденсации изатилиденмалононитрилов, малононитрила и 2-тиофенметиламина (или фурфуриламина) получены соответствующие производные спиропиридина. Кроме того, взаимодействием бензилиденмалононитрилов с тиосемикарбазидом или 2,4-динитрофенилгидразином получаются соответствующие Шиффовы основания. Структуры полученных соединений подтверждены методами ${ }^{1} \mathrm{H} u{ }^{13} \mathrm{C}$ ЯМР спектроскопии. Ключевые слова: изатилиденмалононитрил, малононитрил, амины, тиосемикарбазид, гидразин.
\end{abstract}

Received 21.02.2018. 